\title{
The Community Health Assessment Program in the Philippines (CHAP-P) diabetes health promotion program for low- to middle- income countries: study protocol for a cluster randomized controlled trial
}

\author{
Gina Agarwal ${ }^{* *}$ (D) Ricardo N. Angeles ${ }^{1,4}$, Lisa Dolovich ${ }^{1,2}$, Janusz Kaczorowski ${ }^{3}$, Jessica Gaber ${ }^{1}$, Dale Guenter ${ }^{1}$,
} Floro Dave Arnuco ${ }^{4}$, Hilton Y. Lam ${ }^{5}$, Lehana Thabane ${ }^{6}$, Daria O'Reilly ${ }^{6}$, Rodelin M. Agbulos ${ }^{7}$, Rosemarie S. Arciaga ${ }^{4,8}$, Jerome Barrera ${ }^{4}$, Elgie Gregorio ${ }^{4}$, Servando Halili $\mathrm{Jr}^{9}$, Norvie Jalani ${ }^{10}$ and Fortunato Cristobal ${ }^{4}$

\begin{abstract}
Background: Type 2 diabetes is increasing globally, with the highest burden in low- to middle-income countries (LMICs) such as the Philippines. Developing effective interventions could improve detection, prevention, and treatment of diabetes. The Cardiovascular Health Awareness Program (CHAP), an evidence-based Canadian intervention, may be an appropriate model for LMICs due to its low cost, ease of implementation, and focus on health promotion and disease prevention. The primary aim of this study is to adapt the CHAP model to a Philippine context as the Community Health Assessment Program in the Philippines (CHAP-P) and evaluate the effect of CHAP-P on glycated hemoglobin (HbA1c) compared to a random sample of community residents in control communities.

Methods: Six-month, 26-community (13 intervention, 13 control) parallel cluster randomized controlled trial in Zamboanga Peninsula, an Administrative Region in the southern Philippines. Criteria for community selection include: adequate political stability, connection with local champions, travel feasibility, and refrigerated space for materials. The community-based intervention, CHAP-P sessions, are volunteer-led group sessions with chronic condition assessment, blood pressure monitoring, and health education. Three participant groups will be involved: 1) Random sample of community participants aged 40 or older, 100 per community (1300 control, 1300 intervention participants total); 2) Community members aged 40 years or older who attended at least one CHAP-P session; 3) Community health workers and staff facilitating sessions. Primary outcome: mean difference in $\mathrm{HbA} 1 \mathrm{c}$ at 6 months in intervention group individuals compared to control. Secondary outcomes: modifiable risk factors, health utilization and access (individual); diabetes detection and management (cluster). Evaluation also includes community process evaluation and cost-effectiveness analysis.

(Continued on next page)
\end{abstract}

\footnotetext{
* Correspondence: gina.agarwal@gmail.com

'Department of Family Medicine, McMaster University, 1280 Main St W,

Hamilton, Ontario L8S 4L8, Canada

Full list of author information is available at the end of the article
}

(c) The Author(s). 2019 Open Access This article is distributed under the terms of the Creative Commons Attribution 4.0 International License (http://creativecommons.org/licenses/by/4.0/), which permits unrestricted use, distribution, and reproduction in any medium, provided you give appropriate credit to the original author(s) and the source, provide a link to the Creative Commons license, and indicate if changes were made. The Creative Commons Public Domain Dedication waiver (http://creativecommons.org/publicdomain/zero/1.0/) applies to the data made available in this article, unless otherwise stated. 
(Continued from previous page)

Discussion: CHAP has been shown to be effective in a Canadian setting. Individual components of CHAP-P have been piloted locally and shown to be acceptable and feasible. This study will improve understanding of how best to adapt this model to an LMIC setting, in order to maximize prevention, detection, and management of diabetes. Results may inform policy and practice in the Philippines and have the potential to be applied to other LMICs.

Trial registration: ClinicalTrials.gov (NCT03481335), registered March 29, 2018.

Keywords: Cluster randomized trial, Low- to middle-income countries (LMICs), Philippines, Diabetes mellitus, Hypertension, Health promotion, Disease prevention

\section{Background}

As of 2013, an estimated 382 million people are living with type 2 diabetes globally, with the large majority of these individuals living in low- to middle-income countries (LMICs) [1]. It is estimated that $45.8 \%$ of individuals with diabetes are undiagnosed, with $83.8 \%$ of those undiagnosed being from LMICs [2]. The number of people with diabetes is expected to increase significantly by 2035, with the projected increase higher in low (108\%) and low-middle income (60\%) countries compared to upper-middle (51\%) and high income (28\%) countries [1]. For LMICs, one of the challenges of managing the impact of diabetes is developing effective and low-cost interventions to prevent or delay the onset of type 2 diabetes that can be successfully implemented, scaled up, and sustained [3]. Several studies have demonstrated the cost-effectiveness of early diagnosis and management of diabetes through the use of opportunistic screening and risk assessment screening tools [4, 5]. Therefore, adopting and testing an effective low-cost intervention that enhances early diagnosis and management of diabetes shows promise for LMICs.

In 2013, the Philippines, considered an LMIC, had an overall diabetes prevalence of $6.0 \%$, an estimated 1.7 million people with undiagnosed diabetes, and 54,535 diabetes-related deaths [6]. The Philippines is estimated to have over 6 million people with diabetes by the year 2035 [1]. The Philippines has begun implementation of the World Health Organization Package of Essential Noncommunicable Disease Interventions for primary care settings in low resource areas [7], created the National Centre for Disease Prevention and Control in 2000 including a diabetes-specific office, and has listed reduction of mortality and morbidity from lifestyle-related diseases such as diabetes as one of the goals in the "National Objectives for Health 2005-2010" [8]. However, there are currently some gaps in the areas of detection and treatment for diabetes. For example, though screening kits and medications are available at no cost, the case detection for new cases of diabetes is poor, the diabetes registry is poorly maintained, and the medications often get left unused in the stockrooms of local health centres (Dr M.A. Mabolo, Philippine Department of Health, personal communication, June 16, 2014). Knowledge about diabetes is also a gap in the Philippines; in a study in one region of the Philippines the mean score for diabetes knowledge among people diagnosed with diabetes was only 43\% [9].

The Cardiovascular Health Awareness Program (CHAP) intervention model may be particularly suited to LMICs due to its low cost, implementability, and focus on population-based health promotion and disease prevention. CHAP is a community-based, primary care-centred, volunteer-led, free of charge, cardiovascular disease risk assessment and blood pressure monitoring program, which is combined with health education sessions for community-dwelling older adults [10]. A large community cluster randomized controlled trial in Canada demonstrated that the CHAP intervention resulted in a statistically significant $9 \%$ reduction in annual hospital admissions due to stroke, heart failure, and heart attacks in people aged 65 and over at the population [11]. The CHAP has been successfully expanded to include a diabetes risk assessment component in the Community Health Awareness of Diabetes (CHAD) program and other community adaptations $[12,13]$.

The Community Health Assessment Program for the Philippines (CHAP-P) was based on a formal partnership between universities in the Philippines (Ateneo de Zamboanga University School of Medicine) and Canada (McMaster University Department of Family Medicine) with guidance from a Project Advisory Committee composed of collaborators and researchers from Canada, the Philippines, Peru, Thailand, Tunisia, and the UK. The CHAP-P intervention was developed through a multi-stage study that combined specific elements of the CHAP and CHAD, adapting the intervention to be more appropriate for LMICs in general and the local setting of communities in Southwestern Philippines (Zamboanga Peninsula) in particular. Zamboanga Peninsula was chosen as the program site for this initiative because it exemplifies underprivileged regions in many LMICs in terms of geographical 
isolation, poverty, and scarce health resources where low-cost, community-owned health programs - such as CHAP-P - are urgently needed.

This multi-stage research project is culminating in a parallel cluster randomized controlled trial (RCT), the protocol of which is the focus of this paper. The primary aim of this RCT is to determine the effects of the CHAP-P intervention on HbA1c levels among a random sample of community residents 40 years of age and older, compared to a random sample of community residents in control communities under usual care. The secondary aims are to determine CHAP-P's effectiveness compared to usual care in impacting: i) modifiable lifestyle risk factors for developing type 2 diabetes; ii) self-reported health utilization and access to care; iii) diabetes detection and management indicators in clusters (screening rates, initiation of medical management, hospital admissions, and mortality due to diabetes and its complications); and iv) program cost-effectiveness and cost-utility.

\section{Theoretical framework and development approach}

The overall project is a three-phase mixed methods evaluation. Phase 1 was a qualitative community scan that examined the sociocultural, economic, and health service context in the Zamboanga Peninsula, Philippines in order to adapt the CHAP intervention for best fit. Phase 2 was a three-stage pilot study to finalize assessment tools and evaluation methods, and identify potential problems with implementation in preparation for the RCT. Finally, Phase 3 is the RCT described in this paper. See Fig. 1 for an overview of the overall project design.

Greenhalgh and colleagues' model of diffusion of innovation [14] was used to guide the adaptation of
CHAP to the Philippines context during Phase 1. Our project is also guided by the Integrated Innovation approach, which posits that one can create a synergistic effect when addressing an issue or challenge by combining social, business, and scientific innovations [15]. Social innovations can bring scientific solutions to a local setting, while business innovations can deliver them at an affordable price point. The Integrated Innovation approach will be combined with the knowledge-to-action process, which depicts the relationship between knowledge creation and action steps to promote the application of knowledge (in our case the generation of CHAP-P) and evaluation of the success of the actions taken [16].

\section{Methods/design \\ Setting}

Zamboanga Peninsula is an Administrative Region of the Philippines on the island of Mindanao in the southern Philippines consisting of three provinces (Zamboanga del Norte, Zamboanga del Sur, and Zamboanga Sibugay) and two independent cities (Isabela and Zamboanga City). In 2012, this region had the fourth highest poverty incidence of the 17 regions of the Philippines (33.7\%) [17]. Communities are separated by sea and mountains, with several ethnic and linguistic groups spread throughout the region and connected by inconsistent transportation and communication systems. The provinces and cities are broken down into municipalities, and the municipalities are broken into the smallest administrative districts in the Philippines, known as barangays, which are small villages or neighbourhoods. Each municipality is classified with an income class ranging from first (the highest income class) to sixth (the lowest)

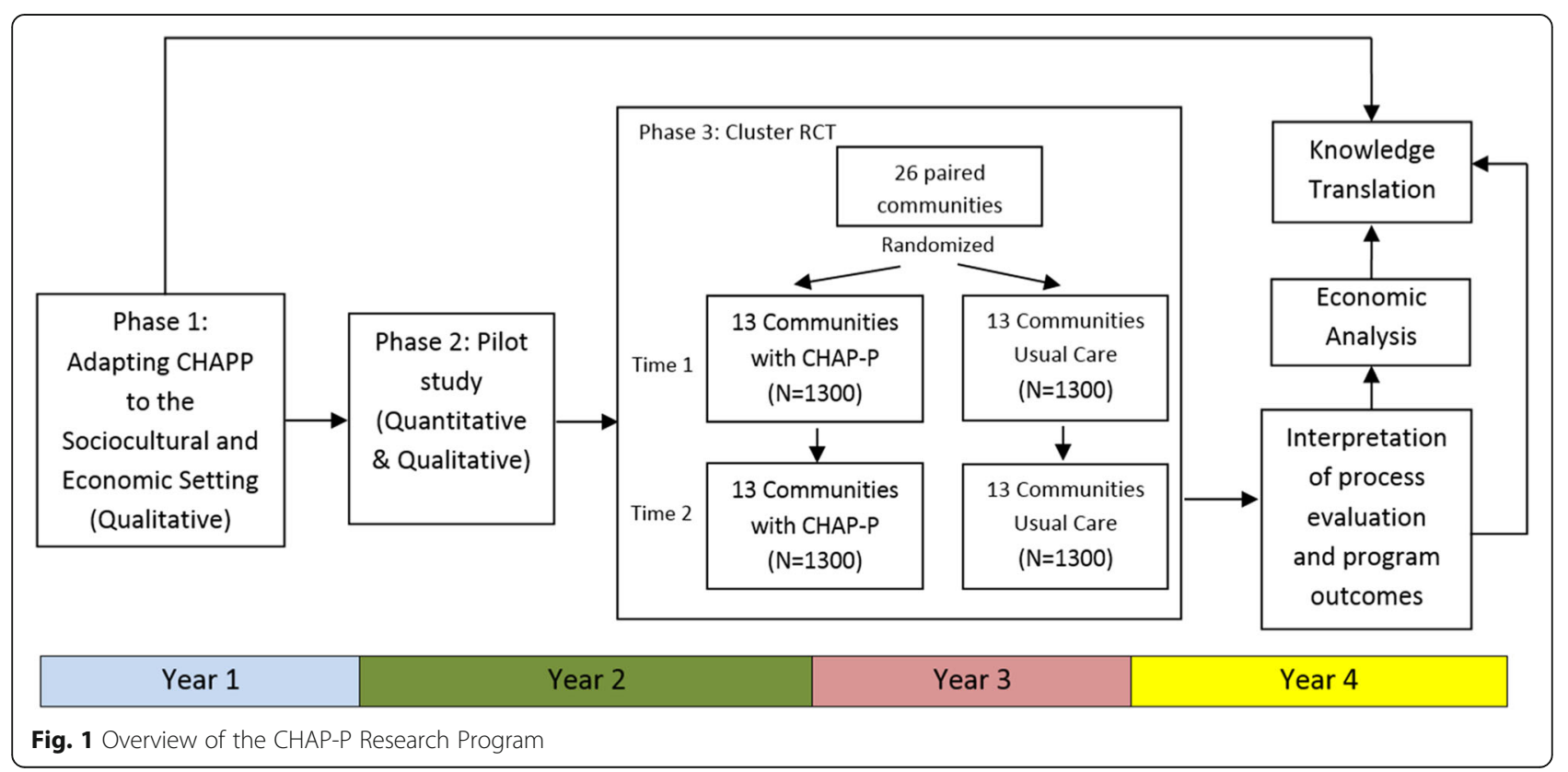


based on their average income in a four-year period. For the evaluation purposes, we will be recruiting 100 randomly selected residents aged 40 and over from each of 26 barangays (communities).

\section{Design}

This study is a 26-community parallel open-label cluster randomized controlled trial. A community cluster design was chosen as CHAP-P is intended as a community-level intervention. Potential communities will be stratified by province, population size, income class, and type (urban versus rural). Thirteen barangays will receive the CHAP-P sessions and will be considered intervention communities, while the other 13 communities will receive care as usual and will be considered control communities. This will be done through paired randomization with staggered starts. Communities will be selected and randomized by the local program manager. Criteria for community selection include: security, connection with a local champion, feasibility for travel, and facility with refrigerated space for the HbA1c kits. Barangays will be paired based on municipality, population size, and similarity in the setting (i.e., religion, being under the same health district providing health services, and distance from the municipal centre). This will be done based on the advice of the local health workers and leaders in the municipalities and cities.
Allocation will be done by computer generated randomization. Measurements of individual participants will be taken as a repeated cross-sectional sample at baseline and at 6 months. The Pragmatic-Explanatory Continuum Indicator Summary 2 (PRECIS-2) [18] was used to make design decisions based on the pragmatism of the trial (see Fig. 2). Reporting will follow the CONSORT 2010 statement: extension for cluster randomized trials [19]; reporting of this protocol follows the SPIRIT 2013 checklist [20], see Additional file 5.

\section{Participant recruitment}

There will be three participant groups in the study. First, 100 participants will be randomly selected and recruited from each of the 26 barangays involved in the trial for a community survey. Households will be chosen via door-to-door systematic random sampling conducted by research staff. Within selected households, individuals 40 years of age or older will be eligible to participate in the survey. If there is more than one eligible and willing individual within a household, the last-birthday selection method [21] will be used to choose a single individual to participate. If there are no eligible or willing participants within a household, the team will move on to the next household based on the systematic random sampling procedure. A member of the research staff team will

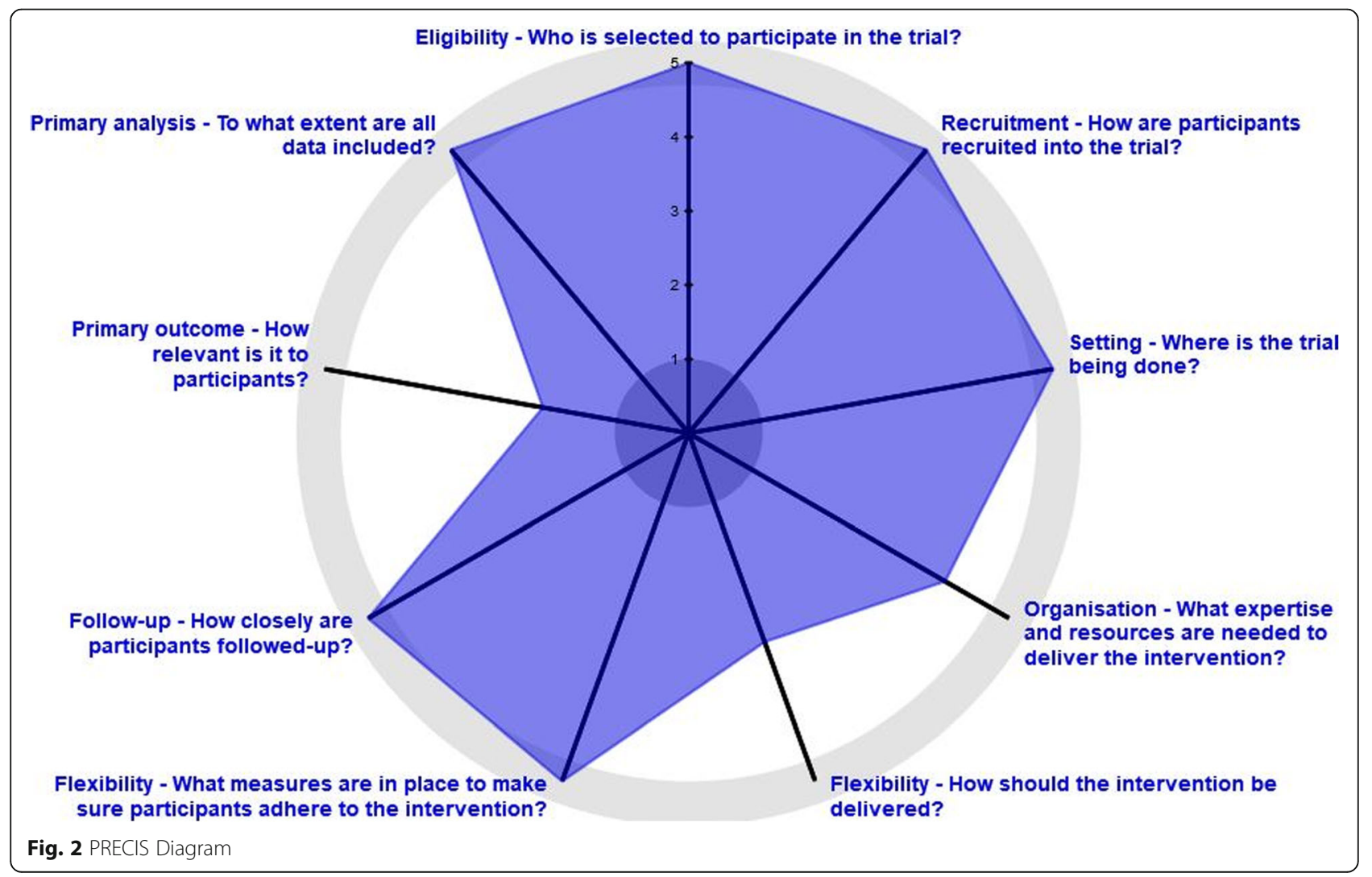


ask individuals selected to participate in the study to provide consent, and the survey will be conducted with consenting individuals (See Additional files 1, 2, 3 and 4 for all consent forms). Participants will then be asked to go to a community location for the $\mathrm{HbA1c}$ test and to be provided with a small token of appreciation. The survey participants are not necessarily the same individuals who will attend the CHAP-P sessions in intervention communities, though entire communities will be invited to the CHAP-P sessions.

The second participant group are those that attend the CHAP-P sessions. CHAP-P participants are community residents aged 40 years of age or older. As part of participating in the CHAP-P sessions, individuals give consent to participate in the study and for the information collected during the sessions to be linked with municipal/city health office records.

The final participant group is the Barangay Health Workers (BHWs) and other Lead Local Organization (LLO) staff that will be involved in facilitating the CHAP-P sessions. They will be recruited through convenience sampling with research team members inviting those who have been involved with the CHAP-P intervention. Those BHWs and other LLO staff that consent to be part of the evaluation will be invited to participate in focus groups or key informant interviews, depending on their role and availability.

Due to the nature of the intervention as a community-wide health promotion program, this is an open label trial. However, community survey participants are not necessarily aware of the ongoing trial, community assignments, and study group allocation of their community.

\section{Outcomes}

\section{Primary outcome and measure}

The primary outcome is the mean difference in HbA1c at 6 months in a random sample of individuals from the random sample of individuals from the intervention barangays compared to the control barangays. HbA1c will be tested at a community location at baseline and 6 months, after the participants have completed the community survey in their homes.

\section{Secondary outcomes and measures}

There are a number of secondary outcomes which will evaluate the mean differences between intervention and control groups at 6 months. These outcomes, whether they pertain to cluster or individual participant level, and their measures and sources are listed in Table 1.

\section{Community process evaluation and fidelity checks}

A community process evaluation will also be undertaken during the project in order to monitor the implementation of the CHAP-P intervention to assess for any problems or process issues during or after the implementation of the project. Monthly reports from communities, monthly observational fidelity checklists from research assistants, and qualitative focus groups/interviews will be analyzed for this component of the evaluation.

Table 1 Secondary Outcomes

\begin{tabular}{|c|c|c|}
\hline Outcome & Level & Measure, Source \\
\hline Physical activity & Individual & $\begin{array}{l}\text { International Physical Activity Questionnaire (IPAQ) [22] } \\
\text { and modified CP@clinic survey [13] }\end{array}$ \\
\hline Medication compliance & Individual & Survey questions (self-reported) \\
\hline Blood pressure & Individual & Physical measurements \\
\hline Risk factors for diabetes & Individual & $\begin{array}{l}\text { Finnish Diabetes Risk Score (FINDRISC) [23] on } \\
\text { community survey }\end{array}$ \\
\hline $\begin{array}{l}\text { Risk behaviours for diabetes / cardiovascular issues (activity, diet, smoking, } \\
\text { alcohol, stress) }\end{array}$ & Individual & $\begin{array}{l}\text { Elements of the Health Awareness and Behaviour Tool } \\
\text { (HABiT) on community survey (self-reported) }\end{array}$ \\
\hline Knowledge about diabetes and hypertension & Individual & $\begin{array}{l}\text { Elements of the HABiT on community survey (self- } \\
\text { reported) }\end{array}$ \\
\hline Perceived concern and understanding of risk & Individual & $\begin{array}{l}\text { Elements of the HABiT on community survey (self- } \\
\text { reported) }\end{array}$ \\
\hline Confidence in behaviour change & Individual & $\begin{array}{l}\text { Elements of the HABiT on community survey (self- } \\
\text { reported) }\end{array}$ \\
\hline Number of community residents newly diagnosed with diabetes & Cluster & Rural Health Unit databases \\
\hline aHospital admission rates due to diabetes and diabetes-related conditions, & Cluster & Central Department of Health database \\
\hline
\end{tabular}
tension, myocardial infarction (MI), stroke, congestive heart failure

aMortality rates due to diabetes and diabetes-related conditions, hypertension, Cluster Regional Field Health Surveillance Information Systems $\mathrm{MI}$, stroke, congestive heart failure based on ICD-10 codes

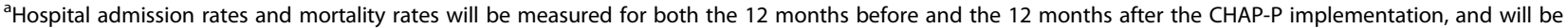
divided by the mid-year population estimates 


\section{Cost-effectiveness and cost-utility}

A cost-effectiveness analysis will be conducted comparing the program cost of implementing CHAP-P and healthcare resource utilization costs to percentage reduction in HbA1c. A cost-utility analysis will also be conducted to determine the cost of the program and healthcare resource utilization costs per quality-adjusted life year (QALY) gained, using the EuroQol-5 dimension-5 level (EQ-5D-5 L) $[24,25]$ as the indicator of quality of life.

\section{Data collection and management}

Community survey participants will be interviewed in their homes by trained research staff at baseline and 6 months. Questionnaires will be completed on paper and later entered into a REDCap [26] database by trained research staff. This survey was adapted from the measure used in the CP@clinic program in Canada [13] and includes questions from other validated questionnaires as well as physical measurements such as blood pressure, height, weight, and waist circumference. The questions include: demographics; knowledge about diabetes and cardiovascular health; risk factors and behaviours, including the Finnish Diabetes Risk Calculator (FINDRISC) [23]; quality of life using the EQ-5D-5 L [24, 25]; perceived confidence; perceived concern and understanding of risk; self-efficacy to improve health behaviours; physical activity using the International Physical Activity Questionnaire (IPAQ) [22]; and health utilization and access. The WatchBP Office Target will be used to measure blood pressure as it was found to show the most reliable results based on our pilot study [27]. This community survey will be completed at baseline and at 6 months and will use an open-cohort design.

After completing the community survey in their homes, these participants will be invited to a community location where they will have their glycated hemoglobin (HbA1c) tested using the A1CNow + point-of-care device, which is certified by the National Glycohemoglobin Standardization Program [28]. HbA1c testing will be conducted at baseline and 6 months, following the community survey at each time point.

Focus group discussions will be held at 6 months for study participants, BHWs, and other LLO staff using a standardized focus group guide consisting of open-ended questions primarily focused on identifying the barriers and facilitators to implementing CHAP-P. Further sources of data include monthly community reports from the communities, monthly observational checklists by research assistants, and record review of the CHAP-P session databases and Rural Health Unit databases.

For CHAP-P session participants, the FINDRISC, blood pressure, and other physical measurement data collected as part of the CHAP-P sessions will be included in the evaluation.

Paper data will be stored in a locked cabinet in a locked institutional office. Electronic data will be stored in an encrypted program (REDCap) or in password-protected files on a secure institutional network. Study data will be anonymized. For the community survey and CHAP-P session data, after the full data set is collected (including the HbA1c test results), data will be anonymized. For the qualitative data, once transcripts are produced from the interviews and focus groups, identifiers of participants will be removed.

\section{Intervention}

The 13 intervention barangays will receive the CHAP-P sessions. The intervention will occur as follows (see Fig. 3). First, residents will be invited to attend the CHAP-P sessions, which are facilitated by Barangay Health Workers (BHWs), who are trained local volunteers. The BHW role is a voluntary position accredited by local health boards, which provides primary health care service to the barangays [29]. BHWs will receive program-specific in-person training at the start of CHAP-P, with refresher sessions during their work with the program. During the CHAP-P sessions, the BHWs will collect participants' consent, measure blood pressure, collect other physical measurements (height, weight, waist circumference), and collect participant information to determine clients' risk of diabetes using the FINDRISC [23]. All data will be collected through an electronic REDCap mobile app database [26] via a tablet computer, which was found to be the most accurate and acceptable choice of data collection method for BHWs during the pilot stages [30].

Based on findings during the assessment, BHWs will educate the CHAP-P participants on diabetes, cardiovascular risk factors, and healthy lifestyles, providing educational materials that have been adapted for a local context (including materials such as pamphlets, comic strips, and videos). Those CHAP-P participants whose FINDRISC scores indicate diabetes risk (moderate: score of 12-14, high: score of $15-20$, or very high: score of > 20) will be referred to the Rural Health Unit for fasting blood glucose testing. Those who have blood pressure greater than $140 / 80 \mathrm{mmHg}$ will be referred to the midwife, with those with blood pressure over 180/100 $\mathrm{mmHg}$ being immediately referred to the Rural Health Unit or District Hospital, and those with blood pressure under $180 / 100 \mathrm{mmHg}$ being referred to the Rural Health Unit with less immediacy, for further evaluation and management. Those with other specific risk factors such as low physical activity, high salt intake, or smoking will be given further health education and referred to appropriate local programs. CHAP-P sessions will continue to 


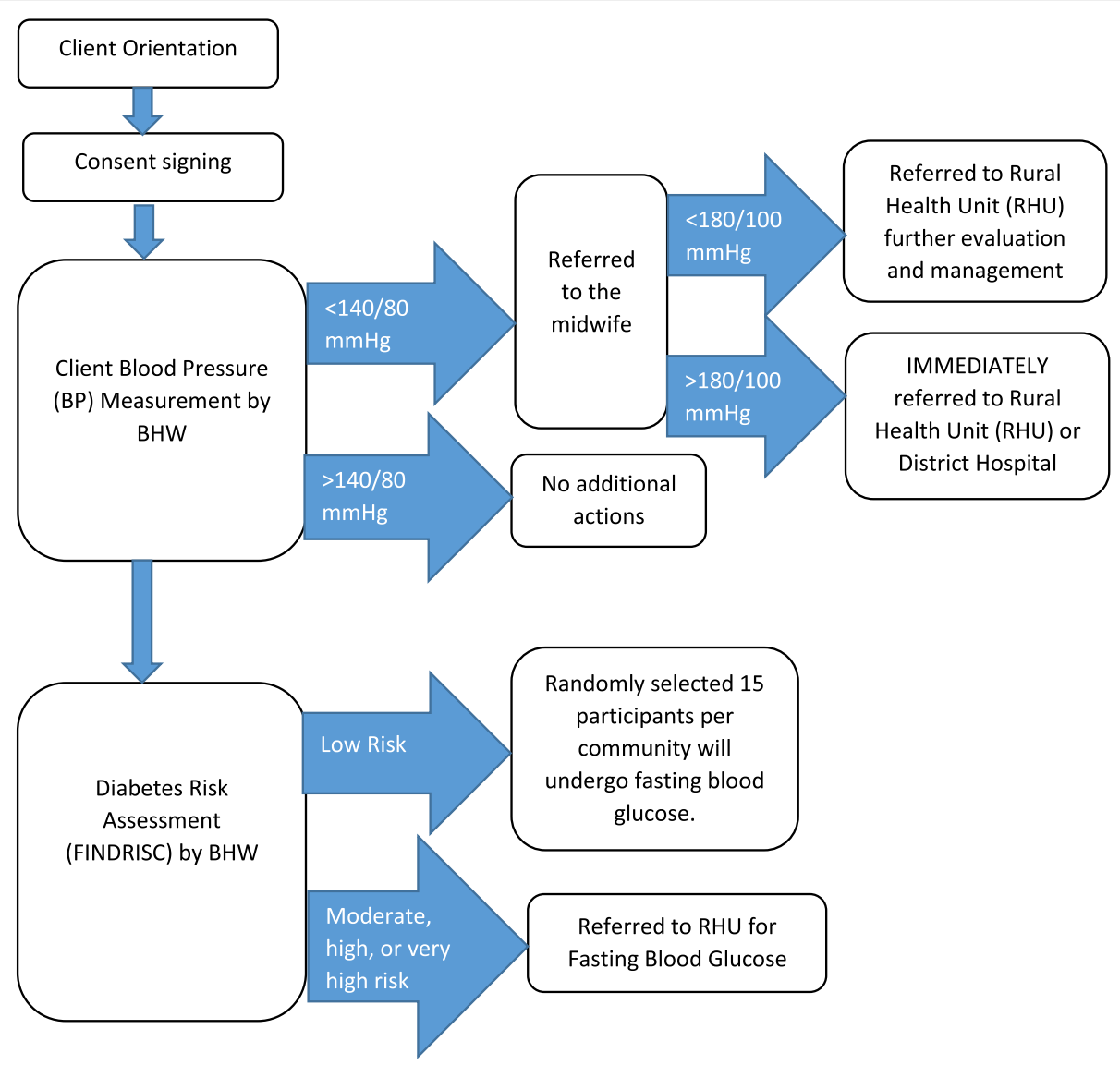

Fig. 3 CHAP-P Intervention

be held twice a month in intervention communities, and residents will be encouraged to continue attending for ongoing follow-up and monitoring. The monthly observational fidelity checklists from research assistants will be undertaken to improve adherence to intervention protocols.

\section{Data analysis}

\section{Quantitative outcomes}

The baseline characteristics will be analyzed using descriptive statistics reported by group as mean (standard deviation) or median (first quartile, third quartile) for continuous variables, and count (percentage) for categorical variables. The analysis of all outcomes to compare the groups will follow intention-to-treat principle. We will use multiple imputation to handle missing data. We will use Generalized Estimating Equations (GEE) to make comparisons between intervention and control communities, assuming an exchangeable correlation structure [31]. GEE will allow us to model the correlation of outcomes within communities. All results will be reported as estimates of effect, corresponding 95\% confidence interval and associated $p$-values. All $\mathrm{p}$-values will be reported to three decimal places with those less than 0.001 reported as $p<0.001$. All analyses will be performed using SAS 9.4 (Cary, NC) or Stata 11 (College Station, TX). See Table 2 for the statistical analysis plan.

\section{Qualitative analysis}

Transcripts will be cleaned and data will be summarized using thematic analysis (open coding axial coding, selective coding). QSR International NVivo 11 qualitative analysis software [32], will be used to store and manage qualitative data.

\section{Economic analysis}

Percentage decrease in participant HbAlc will be the measure of effectiveness used in the cost-effectiveness analysis. Cost per QALY will be calculated as the cost-utility measure; QALYs will be computed based on local EQ-5D-5 L values. Both cost-effectiveness and cost-utility analyses will include overall program cost measures and participant health resource utilization and cost thereof. 


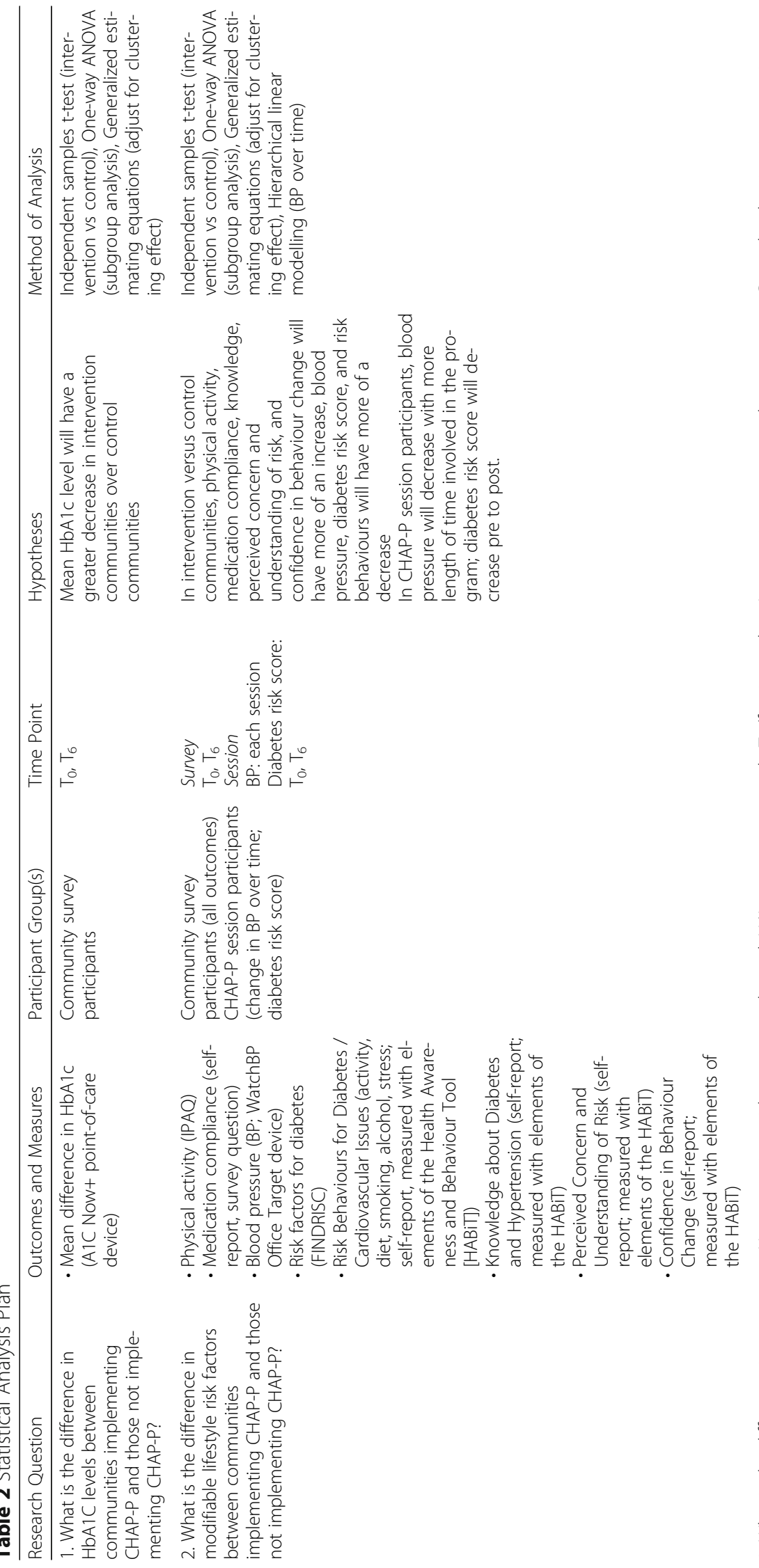

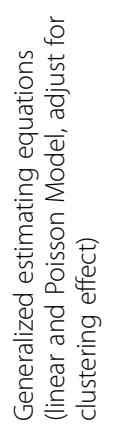

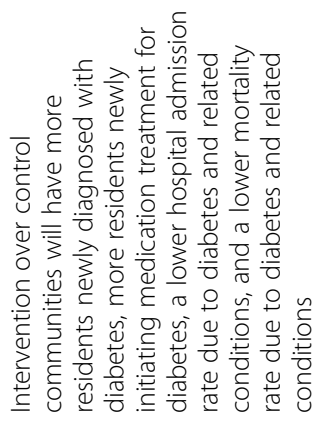

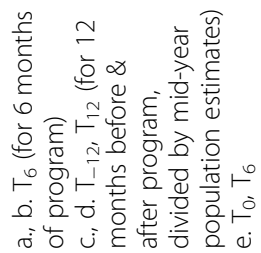

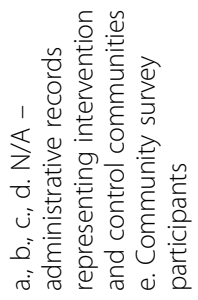

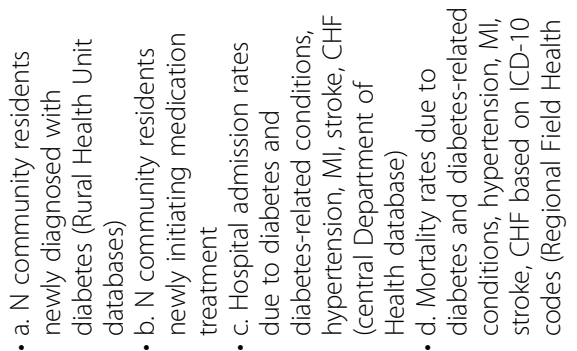

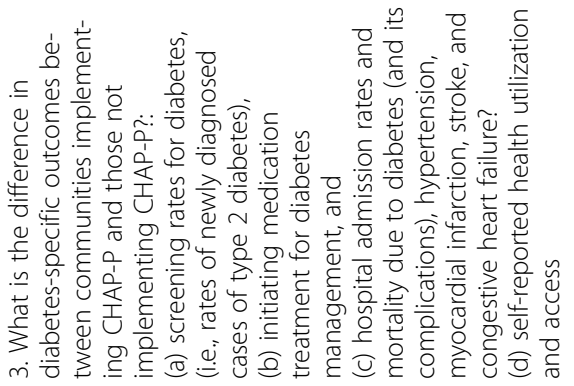




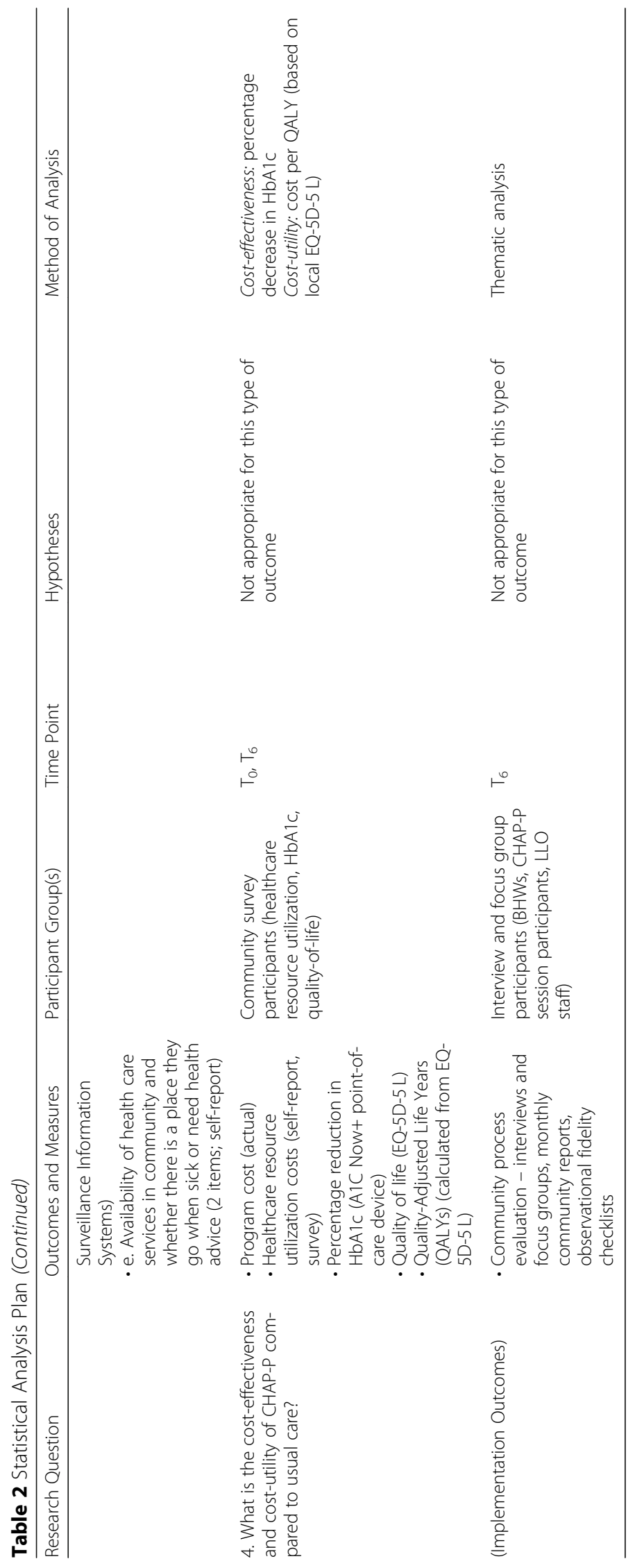




\section{Power and sample size}

CHAP-P is a community-wide intervention and community sizes vary from 3000 to 20,000 residents. We are using paired randomization, therefore intervention and control community pairs need to have relatively similar sizes. Our sample size for individuals was calculated based on a mean difference of HBA1c of $0.2 \%$ (SD = 1.09) with standard parameters (alpha $=0.05$, power $=$ 0.80). This required a sample size of 520 per arm. Based on our pilot, the intraclass correlation coefficient (ICC) was 0.006 . We increased our ICC to 0.01 which inflated our sample size to 1034 per arm. We have opted to take 26 (13 pairs of intervention: control) communities with 100 residents per community giving a total sample size of 2600 residents (1300 per arm).

\section{Discussion}

This study will provide a robust evaluation of a community diabetes program in the Philippines. Though the country is committed to preventing and treating lifestyle-related diseases such as diabetes, the level of commitment to implement programs and the amount of diabetes-related activities already being implemented varies greatly among communities [8]. Some community-based programs for type 2 diabetes tested in other LMICs including elements such as educational sessions, lifestyle instruction, and self-monitoring have shown significant positive outcomes including lowering weight, waist circumference, fasting plasma glucose levels, and HbA1c [33, 34]. Diabetes self-management programs and other community-based interventions are being implemented across the Philippines, yet large-scale experimental studies are still a gap in the literature [9, 35-37]. This study will help fill that gap.

A major strength of this study is the multi-phase, multi-year nature of the overall research program. The intervention was based on the evidence-based CHAP model from Canada, though needed to be adapted for the local context. Phase 1, the qualitative community scan, and Phase 2, piloting the elements, were vital to integrate the components and building a diabetes intervention that would make sense in the context of the Zamboanga Peninsula, Philippines. The mixed methods design included throughout the research program is another strength, with qualitative data included in this $\mathrm{RCT}$ to help explain and interpret quantitative results. Initially, a stepped-wedge cluster RCT was planned rather than a parallel cluster RCT, yet instead of solving issues of logistical constraints [38], it introduced some the stepped-wedge design would have been more expensive and more time-consuming. Due to the variation in communities across the Philippines, the focus on a community-level approach is a key element in making a program such as CHAP-P work. The integration of perspectives from multiple key stakeholders throughout the process solidifies communities' buy-in to the project. Finally, the health economics component of this study will provide policymakers and funders the information they need to decide whether implementation of a program such as CHAP-P is cost-effective for their communities.

Though we combined many elements to fit the program to the setting, there are still some limitations that warrant consideration. First, recruitment for CHAP-P sessions can be difficult, particularly within urban areas, and as a community intervention it is important to reach a substantial portion of the community. To improve penetration within the communities, we have decided to ensure the CHAP-P sessions rotate between puroks (a subdivision of a barangay) rather than having them in the same location week-by-week. Second, the mobile HbA1c test kit can be difficult to work with, as it only lasts for approximately 5 minutes in the heat and humidity of the Philippines. Due to that constraint, all HbA1c tests are now completed at a common community location; however, those locations still need refrigeration for the test kits, so access to refrigerated space is an inclusion criterion for communities. Finally, during the pilot studies there were some security concerns in the region that limited travel of the research team, so something similar may occur during the RCT implementation. This may affect the ability of the research team to visit communities for monthly fidelity checks, yet the intervention will be able to continue as BHWs can continue facilitating CHAP-P sessions in their local communities.

This study has the potential to improve diabetes detection, management, and prevention in the Philippines and similar LMICs. The results from this study will be shared with policymakers (municipal, provincial, regional, national) in the Philippines and our research partners in other LMICs and the Global Alliance for Chronic Diseases. Results will also be published in relevant conference and journals to disseminate our findings to other researchers and policy makers planning to implement an out-of-the-box program for diabetes detection, management, and prevention.

\section{Additional files}

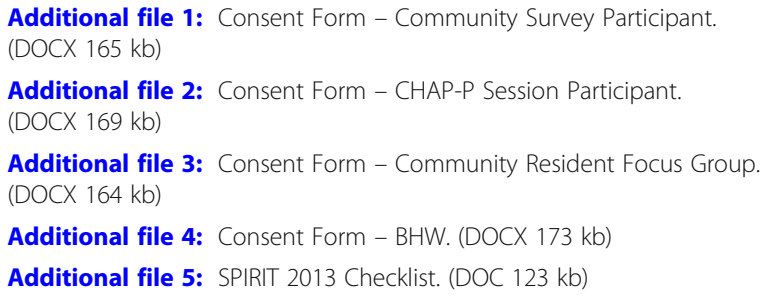


CHAP: Cardiovascular Health Awareness Program; CHAP-P: Community Health Assessment Program in the Philippines; CIHR: Canadian Institutes of Health Research; EQ-5D-5 L: EuroQol-5 dimension-5 level (quality of life measure); FINDRISC: Finnish Diabetes Risk Score (measure); GACD: Global Alliance of Chronic Diseases; HABiT: Health Awareness and Behaviour Tool; HbA1c/ A1C: Glycated hemoglobin; HiREB: Hamilton Integrated Research Ethics Board, Canada; IDRC: International Development Research Centre; IPAQ : International Physical Activity Questionnaire (measure); LMIC: Low- to middle-income country; QALY: Quality-adjusted life year; RCT: Randomized controlled trial

\section{Acknowledgements}

The authors would like to thank the entire project team for their contributions to this research program, including staff and stakeholders in the Philippines and Canada, and the members of our international Project Advisory Group (including 5 primary investigators, 6 Canadian coinvestigators or advisors, 5 Filipino co-investigators, a GACD representative, Canadian and Filipino research staff, and 4 other advisors from Tunisia, Thailand, and Peru).

\section{Funding}

This project is funded through the Canadian Institutes of Health Research (CIHR; project \#139438) and the International Development Research Centre (IDRC; project \#107826-001) via the Global Alliance of Chronic Diseases (GACD; project DM04). The funding bodies had no role in study design, collection, analysis, interpretation of data, or in writing the manuscript.

\section{Availability of data and materials}

Not applicable.

\section{Authors' contributions}

GA and FC are leading the project for Canada and the Philippines, respectively. $G A, F C, R N A, D G, L D$, and JK contributed to the development of the research design and methods. RSA, RMA, JB, EG, SH, FDA, and NJ contributed to the contextualization of the research design and methods to the local setting. HYL and $\mathrm{DO}$ are contributing to the development of the methods for economic analysis. LT is contributing to the methodology for statistical analysis. JG contributed to drafting of the article, acquisition of ethical approval, and registration to Clinical Trials with GA and RNA. All authors read and approved the final manuscript.

\section{Ethics approval and consent to participate}

This trial and all materials (consent, database, education materials) has obtained ethics approval from the Ateneo de Zamboanga (ADZU) research ethics board, Philippines (approval \#2018-SM-0001), and the Hamilton Integrated Research Ethics Board (HiREB), Canada (project ID \#4303). Any amendments regarding protocol changes will be submitted to both ethical review boards. The trial is also registered with ClinicalTrials.gov (identifier NCT03481335, first posted March 29, 2018; last updated August 17, 2018). Signed, written, informed consent will be obtained from study participants in all groups prior to participation in elements of the study. To maintain confidentiality, data will be de-identified prior to analysis. The data that is collected will only be made available for review by authorized persons including CHAP-P research team in the Philippines and Canada, institutional review boards, and transcriber of the focus groups. Information stored on computers will be protected by a password. Information stored on paper will be kept in locked cabinets.

\section{Consent for publication}

Not applicable.

\section{Competing interests}

The authors declare that they have no competing interests.

\section{Publisher's Note}

Springer Nature remains neutral with regard to jurisdictional claims in published maps and institutional affiliations.

\section{Author details}

${ }^{1}$ Department of Family Medicine, McMaster University, 1280 Main St W, Hamilton, Ontario L8S 4L8, Canada. '2Leslie Dan Faculty of Pharmacy,
University of Toronto, 144 College Street, Toronto, Ontario M5S 3M2, Canada. ${ }^{3}$ Department of Family and Emergency Medicine, University of Montreal and CRCHUM, 850 Saint-Denis St, Montreal, Quebec H2X 0A9, Canada. ${ }^{4}$ School of Medicine, Ateneo de Zamboanga University, La Purisima St, 7000

Zamboanga City, Philippines. ${ }^{5}$ Institute of Health Policy and Development Studies, University of the Philippines Manila, One Adriatico Place Tower One, Adriatico Street Corner Pedro Gill Street, 1000 Manila, Philippines. ${ }^{6}$ Department of Health Research Methods, Evidence, and Impact, McMaster University, 1280 Main St W, Hamilton, Ontario L8S 4L8, Canada. ${ }^{7}$ Zamboanga City Health Office, Pettit Barracks, 7000 Zamboanga City, Philippines.

${ }^{8}$ Zamboanga Medical Research Foundation, Zamboanga City, Philippines.

${ }^{9}$ Graduate School, Ateneo de Zamboanga University, La Purisima St, 7000

Zamboanga City, Philippines. ${ }^{10}$ Department of Health, Zamboanga Peninsula, 7000 Zamboanga City, Philippines.

Received: 27 February 2019 Accepted: 14 May 2019

Published online: 03 June 2019

\section{References}

1. Guariguata L, Whiting DR, Hambleton I, Beagley J, Linnenkamp U, Shaw JE. Global estimates of diabetes prevalence for 2013 and projections for 2035. Diabetes Res Clin Pract. 2014;103:137-49.

2. Beagley J, Guariguata L, Weil C, Motala AA. Global estimates of undiagnosed diabetes in adults. Diabetes Res Clin Pract. 2014;103:150-60.

3. Narayan KM, Chan J, Mohan V. Early identification of type 2 diabetes: policy should be aligned with health systems strengthening. Diabetes Care. 2011; 34:244-6.

4. CDC Diabetes Cost-Effectiveness Study Group. The cost-effectiveness of screening for type 2 diabetes. JAMA. 1998;280:1757-63.

5. Gillies CL, Lambert PC, Abrams KR, Sutton AJ, Cooper NJ, Hsu RT, Davies MJ, Khunti K. Different strategies for screening and prevention of type 2 diabetes in adults: cost effectiveness analysis. BMJ. 2008;336:1180-5.

6. International diabetes federation. Global diabetes scorecard: tracking Progress for action. Brussels: International diabetes federation; 2014.

7. Package of essential noncommunicable (PEN) disease interventions for primary health Care in low-resource Settings. http://www.who.int/ cardiovascular diseases/publications/pen2010/en/. Accessed 8 Feb 2019.

8. Beran D, Higuchi M. Delivering diabetes Care in the Philippines and Vietnam: policy and practice issues. Asia Pac J Public Health. 2013;25: 92-101.

9. Ardena GJRA, Paz-Pacheco E, Jimeno CA, Lantion-Ang FL, Paterno E, Juban $\mathrm{N}$. Knowledge, attitudes and practices of persons with type 2 diabetes in a rural community: phase I of the community-based diabetes selfmanagement education (DSME) program in San Juan, Batangas, Philippines. Diabetes Res Clin Pract. 2010;90:160-6.

10. Kaczorowski J, Chambers LW, Karwalajtys T, Dolovich L, Farrell B, McDonough B, Sebaldt R, Levitt C, Hogg W, Thabane L, et al. Cardiovascular health awareness program (CHAP): a community cluster-randomised trial among elderly Canadians. Prev Med. 2008:46:537-44.

11. Kaczorowski J, Chambers LW, Dolovich L, Paterson JM, Karwalajtys T, Gierman T, Farrell B, McDonough B, Thabane L, Tu K, et al. Improving cardiovascular health at population level: 39 community cluster randomised trial of cardiovascular health awareness program (CHAP). BMJ. 2011;342: $\mathrm{d} 442$.

12. Agarwal G. Primary care diabetes: issues facing family doctors. (Unpublished PhD thesis). McMaster University; 2011.

13. Agarwal G, Angeles R, Pirrie M, Marzanek F, McLeod B, Parascandalo J, Dolovich L. Effectiveness of a community paramedic-led health assessment and education initiative in a seniors' residence building: the community health assessment program through emergency medical services (CHAPEMS). BMC Emerg Med. 2016;17:8.

14. Greenhalgh T, Robert G, Macfarlane F, Bate P, Kyriakidou O. Diffusion of innovations in service organizations: systematic review and recommendations. Milbank Q. 2004;82:581-629.

15. Grand Challenges Canada/grand Défis Canada: Integrated innovation. 2010.

16. Graham ID, Logan J, Harrison MB, Straus SE, Tetroe J, Caswell W, Robinson N. Lost in knowledge translation: time for a map? J Contin Educ Heal Prof. 2006;26:13-24.

17. National Statistical Coordination Board: 2012 Full Year Official Poverty Statistics of the Philippines 2013. 
18. Loudon K, Treweek S, Sullivan F, Donnan P, Thorpe KE, Zwarenstein M. The PRECIS-2 tool: designing trials that are fit for purpose. BMJ. 2015;350:h2147.

19. Campbell MK, Piaggio G, Elbourne DR, Altman DG, Group C. Consort 2010 statement: extension to cluster randomised trials. BMJ. 2012;345:e5661.

20. Chan AW, Tetzlaff JM, Altman DG, Laupacis A, Gotzsche PC, Krle AJK, Hrobjartsson A, Mann H, Dickersin K, Berlin JA, et al. SPIRIT 2013 statement: defining standard protocol items for clinical trials. Rev Panam Salud Publica. 2015;38:506-14.

21. Gaziano C. Last-birthday selection. In: Lavrakas PJ, editor. Encyclopedia of Survey Research Methods. Thousand Oaks: Sage Publications, Inc.; 2011.

22. Craig CL, Marshall AL, Sjostrom M, Bauman AE, Booth ML, Ainsworth BE, Pratt M, Ekelund U, Yngve A, Sallis JF, Oja P. International physical activity questionnaire: 12-country reliability and validity. Med Sci Sports Exerc. 2003; 35:1381-95.

23. Lindstrom J, Tuomilehto J. The diabetes risk score: a practical tool to predict type 2 diabetes risk. Diabetes Care. 2003;26:725-31.

24. Brooks R. EuroQol: the current state of play. Health Policy. 1996;37:53-72.

25. EuroQol G. EuroQol--a new facility for the measurement of health-related quality of life. Health Policy. 1990;16:199-208.

26. Harris PA, Taylor R, Thielke R, Payne J, Gonzalez N, Conde JG. Research electronic data capture (REDCap)--a metadata-driven methodology and workflow process for providing translational research informatics support. J Biomed Inform. 2009;42:377-81.

27. Guenter D, Angeles R, Kaczorowski J, Agarwal G, Cristobal FL, Arciaga R, Smith JF, Kessomboon P, Jarraya F, Agbulos R, et al. Choosing the optimal method of blood pressure measurement for limited-resource rural communities in the "community health assessment program-Philippines". Clin Hypertens (Greenwich). 2017;19(9):899-903.

28. Little RR, Rohlfing CL, Wiedmeyer HM, Myers GL, Sacks DB, Goldstein DE. Committee NS: the national glycohemoglobin standardization program: a five-year progress report. Clin Chem. 2001;47:1985-92.

29. Department of Health (DOH): What is barangay health worker?: Republic of the Philippines Department of Health (DOH). https://www.doh.gov.ph/ node/837. Accessed 8 Feb 2019.

30. Kaczorowski J, Dolovich L, Agarwal G, Guenter D, Angeles R, Arciaga R, Agbulos R, Cristobal FL. Feasibility and Accuracy of Screening Tools for Diabetes and Hypertension in Municipalities the Zamboanga Peninsula, Philippines. Presented at the North American Primary Care Group (NAPCRG) Annual Meeting. Montreal; 2017.

31. Hardin JW, Hilbe JM. Generalized Estimating Equations. Boca Raton: CRC Press; 2002.

32. NVivo qualitative data analysis software 11 edition [Computer software]. QSR international Pty Itd. 2015.

33. $\mathrm{Hu} \mathrm{Z}$, Qin $\mathrm{LL}, \mathrm{Xu} \mathrm{HL}$. One-year results of a synthetic intervention model for the primary prevention of T2D among elderly individuals with prediabetes in rural China. Int J Environ Res Public Health. 2017;14. https://doi.org/10. 3390/ijerph14040417.

34. Pimentel GD, Portero-Mclellan KC, Oliveira EP, Spada APM, Oshiiwa M, Zemdegs JCS, Barbalho SM. Long-term nutrition education reduces several risk factors for type 2 diabetes mellitus in Brazilians with impaired glucose tolerance. Nutr Res. 2010;30:186-90.

35. van Olmen J, Ku GM, van Pelt M, Kalobu JC, Hen H, Darras C, Van Acker K, Villaraza B, Schellevis F, Kegels $G$. The effectiveness of text messages support for diabetes self-management: protocol of the TEXT4DSM study in the democratic republic of Congo, Cambodia and the Philippines. BMC Public Health. 2013;13:423.

36. Ku GM, Kegels $\mathrm{G}$. The performance of the Finnish diabetes risk score, a modified Finnish diabetes risk score and a simplified Finnish diabetes risk score in community-based cross-sectional screening of undiagnosed type 2 diabetes in the Philippines. Prim Care Diabetes. 2013;7:249-59.

37. Ku GMV, Kegels G. Knowledge, attitudes and perceptions of people with type 2 diabetes as related to self-management practices: results of a cross-sectional study conducted in Luzon, Philippines. Chronic Illness. 2015;11:93-107.

38. Hemming K, Haines TP, Chilton PJ, Girling AJ, Lilford RJ. The stepped wedge cluster randomised trial: rationale, design, analysis, and reporting. BMJ. 2015; 350:h391.

\section{Ready to submit your research? Choose BMC and benefit from:}

- fast, convenient online submission

- thorough peer review by experienced researchers in your field

- rapid publication on acceptance

- support for research data, including large and complex data types

- gold Open Access which fosters wider collaboration and increased citations

- maximum visibility for your research: over $100 \mathrm{M}$ website views per year

At $\mathrm{BMC}$, research is always in progress.

Learn more biomedcentral.com/submissions 